\title{
Nicotina y modelos animales: ¿qué nos aporta el paradigma de enriquecimiento ambiental?
}

\section{Nicotine and animal models: what does the environmental enrichment paradigm tell us?}

\author{
Patricia Mesa-Gresa; Asunción Pérez-Martínez; \\ Rosa Redolat-lBorra
}

\author{
Departamento de Psicobiología. Facultad de Psicología. \\ Universitat de València.
}

\section{Resumen}

El Enriquecimiento Ambiental (Environmental Enrichment o EE) es un modelo de alojamiento basada en la estimulación a nivel físico, cognitivo y sensorial de roedores. Esta condición se caracteriza por el alojamiento de un grupo de animales en cajas grandes que contienen distintos objetos como túneles, juguetes y ruedas de correr. El principal objetivo del presente trabajo es plantear los principales argumentos que sugieren que el alojamiento en condiciones de EE puede disminuir la vulnerabilidad a desarrollar adicción a la nicotina y a otras drogas de abuso, así como revisar estudios experimentales recientes relacionados con este área. Con este fin, se presenta una revisión de los principales cambios inducidos por el EE a nivel físico, neurobiológico y conductual y se exponen los resultados obtenidos en diversos estudios recientes que indican que el alojamiento en EE induce cambios neuroquímicos (potenciación del aumento de liberación de dopamina inducida por la nicotina en el córtex cerebral) y conductuales (mayor capacidad para discriminar la presencia de recompensa y disminución de la impulsividad) que apoyarian la hipótesis planteada. En base a estos resultados, cabria proponer el EE como un modelo adecuado para el estudio de la vulnerabilidad a la adicción a diferentes drogas de abuso como la cocaina o la nicotina aunque es necesario realizar más estudios que permitan establecer las bases neurobiológicas de los efectos inducidos por la exposición a ambientes enriquecidos y su posible relación con modificaciones en los sistemas cerebrales de refuerzo.

Palabras clave: Enriquecimiento ambiental, adicción, nicotina, drogas de abuso, ratones.
Enviar correspondencia a:

Rosa Redolat Iborra.

Departamento de Psicobiología. Facultad de Psicología.

Universitat de València. Avda. Blasco lbáñez 21. 46010 Valencia.

E-mail: Rosa.Redolat@uv.es
Key words: Environmental Enrichment, addiction, nicotine, drugs of abuse, mice.
The Environmental Enrichment (EE) paradigm is a housing condition which aims is to provide physical, cognitive and sensorial stimulation to rodents. Animals are housed in larger cages containing inanimate objects such as tunnels, toys and running wheels. The main aim of the current work is to tackle the arguments which suggest that EE may diminish vulnerability to developing addiction to nicotine and other drugs of abuse and to review recent experimental studies performed in relation to this subject. We discuss the major changes induced by EE at physical, neurobiological and behavioral levels and review the results of recent studies which indicate that EE promotes both neurochemical (potentiation of the increase in dopamine release induced by nicotine in the brain cortex) and behavioral changes (increased ability to discriminate the presence of reward and decreased impulsivity), thus supporting the hypothesis put forward. In light of these results, EE can be proposed as a model for the study of vulnerability to addiction to different drugs of abuse, including cocaine and nicotine, though further studies are needed in order to establish the neurobiological implications of the effects of exposure to enriched environments and their possible relationship with changes in brain reward systems. 
U na pregunta que se plantea frecuentemente en diferentes investigaciones es por qué tan solo un porcentaje de sujetos que experimentan con drogas acaban desarrollando adicción (Green et al., 2010). Actualmente, la adicción se considera una enfermedad crónica que da lugar a cambios duraderos en el funcionamiento cerebral que, a su vez, interactúan con factores del ambiente (Puhl, Blum, Acosta-Torres, y Grigson, 2012). En este marco teórico, algunos autores consideran que el paradigma del Enriquecimiento Ambiental en roedores (Environmental Enrichment o EE) podría constituir un modelo enormemente útil para evaluar los efectos del ambiente sobre la adicción (Carroll, Anker, y Perry, 2009; Chauvet, Lardeux, Goldberg, Jaber, y Solinas, 2009; Magalhaes, Summavielle, Tavares, y de Sousa, 2007; Thiel, Pentkowski, Peartree, Painter, y Neisewander, 2010a). Además, un mejor conocimiento del papel que desempeñan las condiciones ambientales en la susceptibilidad individual a desarrollar adicción podría resultar crucial para mejorar las estrategias de prevención y tratamiento. En este sentido, el paradigma del EE ha demostrado tener enorme potencial para la investigación en este ámbito ya que la exposición a un ambiente enriquecido en etapas críticas del desarrollo parece disminuir la propensión a la adicción (El Rawas, Thiriet, Lardeux, Jaber, y Solinas, 2009; Green et al., 2010; Solinas, Thiriet, Chauvet y Jaber, 2010).

Diversos trabajos en roedores han puesto de manifiesto que en función del tipo de alojamiento se pueden inducir efectos diferenciados a nivel físico, neurobiológico y conductual (Nithianantharajah y Hannan, 2006; van Praag, Kempermann, y Gage, 2000; van Praag, 2009). Los tipos de alojamiento más estudiados han sido el aislamiento social o situación de empobrecimiento ambiental, en el que el animal permanece alojado de manera individual en una caja de pequeñas dimensiones (Lopez, Doremus-Fitzwater, y Becker, 2010); la situación estándar o de enriquecimiento social, en la que se alojan de 4 a 6 sujetos en cajas de laboratorio de dimensiones estándar y, por último, el enriquecimiento ambiental que consiste en el alojamiento de un mayor número de animales en cajas más grandes con distintos tipos de objetos que favorecen su estimulación. El paradigma del EE se constituye como un modelo caracterizado por la estimulación de los animales tanto a nivel físico (con ruedas para correr, túneles, plataformas...), como a nivel cognitivo y sensorial mediante diversos objetos inanimados (casitas, juguetes de distintas formas, texturas y colores, materiales para hacer nidos y campanas, entre otros), así como a nivel social (mediante la interacción de los animales en grandes cajas en las que se alojan entre 5 y 12 e incluso hasta 20 en algunos estudios) (Laviola, Hannan, Macri, Solinas, y Jaber, 2008). En última instancia, se trataría de "enriquecer el ambiente para potenciar al cerebro" (Sale, Berardi, y Maffei, 2009). Esta complejidad proporciona también estimulación a nivel visual, somatosensorial e incluso olfatorio (Carvalho, Araujo, Farias, Rocha-Bezerra, y Cavalheiro, 2009; Nithianantharajah y Hannan, 2006; Redolat y Mesa-Gresa, 2011). El periodo durante el cual se mantienen los animales en estas condiciones y la edad de los mismos varía en función de los objetivos del estudio, de los análisis y pruebas que se realicen y de las hipótesis que guíen la investigación. Estudios recientes indican que 3-4 semanas de enriquecimiento pueden ser suficientes para observar cambios tanto neurobiológicos como conductuales (Mesa-Gresa, Pérez-Martínez, y Redolat, 2010), aunque para algunas tareas, o para incrementar la duración de los cambios, el periodo necesario podría ser más prolongado (Brenes, Padilla, y Fornaguera, 2009).

Desde las primeras investigaciones realizadas en la década de los sesenta, se han documentado cambios fisiológicos, neurobiológicos y conductuales en roedores como resultado del enriquecimiento de su ambiente (Rosenzweig, 1966). Esta situación de alojamiento da lugar a modificaciones estructurales y funcionales en áreas neurales relacionadas, entre otras, con mejoras en aprendizaje y memoria (Chen, Mao, Zhou, Hu, Wang, y Ma, 2010; Peña, Prunell, Dimitsantos, Nadal, y Escorihuela, 2006; Pizzorusso, Berardi, y Maffei, 2007). A nivel neurobiológico, se ha observado que el EE induce un incremento en el tamaño del cerebro, el grosor cortical, la densidad de los contactos sinápticos, el número de células gliales, el tamaño de los núcleos neuronales así como en la cantidad de conexiones dendríticas (Diamond, 2001; van Praag et al., 2000). El EE también amplía las representaciones en el córtex somatosensorial, mejora el procesamiento de la corteza auditiva y visual, incrementa la plasticidad celular y los niveles de proteínas sinápticas, aumenta la neurogénesis en el giro dentado del hipocampo así como la supervivencia de las nuevas neuronas (Kempermann et al., 2010; Segovia, Del Arco, De Blas, Garrido y Mora, 2010). Sin embargo, no se conocen completamente los mecanismos neurobiológicos relacionados con algunos de estos cambios cerebrales inducidos por la estimulación ambiental (Sale et al., 2009, Nithianantharajah y Hannan, 2009). A nivel conductual, se ha observado una disminución de la respuesta de ansiedad y de estrés, una habituación más rápida a los ambientes nuevos y una mejora significativa del aprendizaje y la memoria de los animales alojados en EE respecto a los alojados en la situación estándar o en aislamiento (Ilin y Richter-Levin, 2009; Magalhaes et al., 2007; Meshi et al., 2006).

Recientemente se han ido proponiendo aplicaciones del EE que abren nuevas posibilidades en la investigación neuroconductual, especialmente en relación con su papel no solo preventivo sino incluso con posibilidades curativas en el campo de la adicción a diferentes drogas de abuso (Solinas et al., 2010). Investigaciones realizadas por los equipos de Marcello Solinas (en la Universidad de Poitiers, Francia) y Henriette Van Praag (en el National Institue of Aging, USA) confirman esta hipótesis, mostrando que el alojamiento en un ambiente enriquecido puede prevenir el desarrollo de la adicción a drogas 0 , en última instancia, convertirse en un tratamiento potencial. Por tanto, el principal objetivo en este editorial es mostrar, desde un punto de vista crítico, argumentos y ejemplos que justifican la utilidad del paradigma de EE como estrategia protectora frente a la adicción a la nicotina y a otras drogas de abuso. Esta aproximación pretende ser útil a los investigadores que trabajan en el área de las adicciones, contribuyendo a ampliar su conocimiento respecto a datos recientes que integran un nuevo enfoque 
que permite abordar, desde la perspectiva de la investigación básica, el papel de los factores ambientales con el fin de diseñar intervenciones preventivas o de intervención frente a la adicción a diferentes drogas de abuso (Green, Cain, Thompson, y Bardo, 2003; Wong, Mill, y Fernandes, 2011). Nuestros trabajos en este ámbito se han centrado especialmente en la adicción a la nicotina, por lo que prestaremos atención especial a esta droga después de presentar un breve resumen de la aplicación de este modelo en los estudios sobre adicción a otras drogas de abuso.

\section{Enriquecimiento ambiental y adicción}

Estudios recientes otorgan un papel central a los factores ambientales como posibles determinantes de la sensibilidad a los efectos reforzantes de las drogas de abuso y, por tanto, de la vulnerabilidad a desarrollar adicción (EI Rawas, Thiriet, Nader, Lardeux, Jaber y Solinas, 2011; Solinas et al., 2010; Thiel, Engelheardt, Hood, Peartree, y Neisewander, 2010b). Existe amplia evidencia experimental acerca de la asociación entre adicción a drogas y experiencias adversas como maltrato infantil, estrés del combate, abuso sexual o estrés laboral (Mesa-Gresa y Moya-Albiol et al., 2011; Wong et al., 2011). Se han realizado diversos estudios, tanto en primates como en roedores, acerca de los efectos negativos de la exposición al estrés a edades tempranas, sugiriendo que ante esta situación se producen modificaciones a nivel fisiológico, neurobiológico y hormonal que podrian llevar a una mayor propensión al consumo de sustancias adictivas (Beauquis, Roig, De Nicola, y Saravia, 2010; Laviola et al., 2008; Schloesser, Lehmann, Martinowich, Manji, y Herkenham, 2010; Thiriet et al., 2008). En investigaciones realizadas en sujetos humanos se ha observado que las experiencias negativas durante los primeros años de vida inducen cambios estructurales y funcionales a nivel cerebral que podrian favorecer el consumo de diferentes drogas de abuso, tanto durante la adolescencia como en la edad adulta (Lee y Hoaken, 2007; Mesa-Gresa y Moya-Albiol, 2011). En cambio, las relaciones familiares positivas parecen disminuir las posibilidades de consumo y podrían prevenir el desarrollo de la adicción; por lo que se podría hipotetizar que un ambiente positivo, especialmente durante los periodos críticos de desarrollo, podria tener efectos protectores y/o curativos frente al consumo de drogas (Laviola et al., 2008; MesaGresa y Moya-Albiol, 2011; Solinas et al., 2010; Wong et al., 2011). La principal dificultad de las investigaciones en sujetos humanos se plantea porque en los estudios retrospectivos resulta difícil aislar el "ambiente enriquecido" de otras variables que pueden afectar al sujeto a lo largo de su vida (Nithianantharajah y Hannan, 2009, 2011). Por ello, se sugiere que la utilización de paradigmas como el EE en roedores podría proporcionar un modelo adecuado para investigar posibles efectos "protectores" de un estilo de vida positivo y activo frente a la adicción (Laviola et al., 2008).

En estudios previos se ha demostrado que el EE disminuye la auto-administración de anfetaminas (Bardo, Klebaur, Valone, y Deaton, 2001; Green, Gehrke, y Bardo, 2002).
Bowling y Bardo (1994) observaron que las ratas criadas en ambientes enriquecidos mostraban un incremento en el efecto estimulante a nivel locomotor y de preferencia de lugar inducido por anfetaminas en comparación con las criadas en aislamiento o en condición social. Posteriormente, Bardo y colaboradores confirmaron que el EE disminuye la sensibilización conductual inducida por anfetaminas (Bardo, Bowling, Rowlett, Manderscheid, Buxton, y Dwoskin, 1995). Sin embargo, Thiriet y colaboradores (2011) evaluaron los posibles efectos beneficiosos del EE en la adicción a metanfetamina, sugiriendo que la exposición a un ambiente enriquecido no disminuye sus efectos reforzantes y activadores $y$, por tanto, el EE no parece tener efectos directos sobre la vulnerabilidad al desarrollo de la adicción a esta sustancia (Thiriet et al., 2011).

Los estudios más amplios sobre relación entre EE y vulnerabilidad a la adicción se han realizado fundamentalmente con cocaína. En investigaciones iniciales realizadas por el grupo de Bezard y colaboradores se demostró que las ratas enriquecidas son menos responsivas a esta droga que las mantenidas en ambientes estándar. Además, los ratones enriquecidos muestran un patrón diferente de expresión de la proteína c-fos, incremento en la expresión de factores neurotróficos (especialmente BDNF) y regulación a la baja en la expresión del transportador de dopamina, principal diana de los psicoestimulantes (Bezard et al., 2003; Solinas, Chauvet, Thiriet, El Rawas, y Jaber, 2008), así como disminución de la reactividad de las neuronas estriatales a los efectos de la cocaína y otras drogas dopaminérgicas (Solinas, Thiriet, El Rawas, Lardeux, y Jaber, 2009; Thiriet et al., 2011). En un estudio reciente, Solinas y colaboradores han evaluado el efecto de la exposición a ambientes enriquecidos en roedores que previamente habian desarrollado adicción a la cocaína utilizando para ello diferentes modelos animales (sensibilización conductual, condicionamiento de preferencia de lugar y reinstauración inducida por la droga de una preferencia de lugar ya extinguida) en base a los cuales sugieren que los efectos del EE pueden no ser solo preventivos sino también "curativos" frente a una adicción ya establecida (Smith et al., 2009; Solinas et al., 2010; Thiriet et al., 2011), conclusiones novedosas que abren interesantes vías de investigación.

Green y colaboradores (2010) han planteado recientemente la hipótesis de que la activación repetida de las vías de la recompensa mediante la novedad, el ejercicio y el contacto social llevan a que las ratas criadas en ambientes enriquecidos sean menos vulnerables a la auto-administración compulsiva de diferentes drogas de abuso. En un estudio experimental, estos autores demostraron que las ratas criadas en $\mathrm{EE}$, en comparación con las "empobrecidas", muestran una disminución en la auto-administración de cocaína pero mayor condicionamiento de preferencia de lugar inducido por esta droga, reducción de las conductas relacionadas con depresión pero incremento de la ansiedad y una menor actividad de la proteína CREB en el Núcleo Accumbens (que se asocia con expresión reducida del BDNF). Por tanto, numerosos resultados experimentales apoyan la hipótesis de que el EE podría tener efectos protectores frente a la adicción a la cocaina, aunque no se conocen totalmente los mecanis- 
mos subyacentes. Más recientemente, se ha evaluado si los efectos beneficiosos del EE que se habian demostrado previamente en relación con la cocaína también se observaban en el caso de la heroína, utilizando el paradigma del condicionamiento de preferencia de lugar (El Rawas et al., 2009).

En relación con el alcohol, diversos estudios también han demostrado la utilidad de las manipulaciones ambientales en modelos animales para estudiar posibilidades de intervención en la adicción a esta sustancia (Rueda, Teixera, Yonamine, Camarini, 2011). Se ha hipotetizado que los niños con Sindrome Alcohólico Fetal presentan mayores alteraciones en su desarrollo debido a que son criados en ambientes de "riesgo", exacerbando el impacto de la exposición prenatal al alcohol. De ahí la necesidad de que estos niños sean criados en ambientes adecuados, con condiciones ambientales estructuradas y enriquecidas. Utilizando modelos animales, se han realizado diferentes intentos para evaluar cómo las manipulaciones experimentales del ambiente postnatal pueden utilizarse para "tratar" los efectos de la exposición fetal al alcohol. Estas aproximaciones incluyen el "handling" (manoseo) postnatal, el EE y el entrenamiento basado en la rehabilitación motora (veáse revisión de Hannigan, 0'learyMoore, \& Berman, 2007). En general, se observa que cuando los roedores son criados en ambientes enriquecidos (a nivel motor, sensorial y social) después de haber estado expuestos al alcohol durante el periodo prenatal, el EE puede mitigar los efectos del alcohol sobre la conducta, confirmando la existencia de plasticidad cerebral.

Aunque todavía no se comprenden bien los cambios moleculares que subyacen a los efectos del EE sobre la adicción (Burrows, McOmish, y Hannan, 2010; Stairs y Bardo, 2009), se sugiere que la exposición a este ambiente podría proporcionar a los sujetos mayor capacidad de discriminar la presencia de recompensa (Grimm et al., 2008). En estudios previos, las ratas enriquecidas muestran un incremento en el condicionamiento tanto de la preferencia como de la aversión a un lugar (Bardo et al., 1995; Smith et al., 2005; Smith, Bryant, y McClean, 2003), así como extinción acelerada del condicionamiento del miedo (Pietropaolo, Feldon, Alleva, Cirulli, y Yee, 2006). Ello indicaría que los animales criados en ambientes enriquecidos muestran mayor capacidad de aprendizaje acerca del significado de los estímulos asociados a la recompensa y el castigo, y de distinguir entre "disponibilidad" y "no disponibilidad" del refuerzo. Durante el proceso de extinción, también parecen aprender más rápidamente que sus acciones no van a ser recompensadas y, por ello, dejan de responder más pronto que los criadas en ambientes empobrecidos (Grimm et al., 2008), aunque la exposición al EE parece disminuir sus impulsividad (Hellemans, Nobrega, y Olmstead, 2005; Perry, Stairs, y Bardo, 2008). Estos cambios podrian ir asociados a una disminución de la vulnerabilidad a las conductas de recaída (Grimm et al., 2008). Son necesarios más estudios para establecer las bases neurobiológicas de los efectos que la exposición a un ambiente enriquecido induce sobre los sistemas de refuerzo, aunque las vías dopaminérgica, mesolímbica y mesocortical, así como el glutamato, han sido implicados (Segovia et al., 2010).

\section{Enriquecimiento ambiental y nicotina}

Uno de los motivos por los que creemos relevante plantear esta aproximación es la ausencia de trabajos recientes que integren información sobre el alojamiento en condiciones de EE y la adicción a la nicotina. Como ha quedado reflejado anteriormente, algunos autores (especialmente el grupo de Marcello Solinas) han destacado la relevancia de este paradigma para abordar estudios a nivel de investigación fundamental que ayuden en la búsqueda de nuevos tratamientos. Sin embargo, la mayoría de trabajos se han centrado en drogas como la heroína o la cocaína y pocos autores han prestado atención al interés que podria tener desarrollar modelos de EE con sustancias como la nicotina. Teniendo en cuenta la carencia de trabajos relativos a esta temática, hemos realizado una búsqueda exhaustiva en la base de datos PubMed todas las investigaciones y artículos que abordaran el tema "environmental enrichment+nicotine", obteniendo únicamente 9 citas de las cuales tan solo 5 estaban directamente relacionadas con el tema que nos ocupa. La mayoría de las investigaciones han tratado de dilucidar los factores genéticos y moleculares implicados en la adicción a la nicotina, pero se ha investigado relativamente poco acerca de cómo los factores ambientales asociados a la plasticidad dependiente de la experiencia pueden influir en la modulación y progreso de la adicción a esta sustancia, así como en el cese de la misma o en la recaída tras un periodo de abstinencia (Arndt et al., 2009; Solinas et al., 2010). Existen numerosas investigaciones, realizadas tanto en modelos clínicos como preclínicos, que muestran los efectos y cambios estructurales y moleculares inducidos por la nicotina, pero no se han investigado de modo tan detallado los cambios mediados por el ambiente en el que se desarrolla el sujeto (Coolon y Cain, 2009).

Al igual que la cocaína, la anfetamina o la heroína, la nicotina presente en el tabaco induce un patrón de conducta adictiva (De Biasi y Dani, 2011). Una de las investigaciones más importantes sobre la relación entre EE y adicción a la nicotina, fue la realizada Zhu y colaboradores acerca de la influencia del EE en la liberación de DA inducida por nicotina en áreas del córtex prefrontal medial y del estriado. Se observó que las condiciones de EE eliminan la respuesta de transporte de DA en el córtex prefrontal medial en el grupo control y potencian el incremento inducido por la nicotina en el transporte de DA en este área pero no en la zona del estriado (Zhu, Bardo, Green, Wedlund, y Dowskin, 2007). En una investigación ya clásica del grupo de Green y colaboradores (2003), se analizó el efecto del EE sobre la actividad motora en ratas SpragueDawley tras la administración repetida de nicotina, sugiriendo que el EE podría reducir los efectos estimulantes de esta droga. En un trabajo posterior se confirmó una disminución de la sensibilización a los efectos hiperactivos locomotores de la nicotina en ratas alojadas en EE frente a las mantenidas en ambiente empobrecido o en aislamiento, observándose además que las ratas alojadas en ambiente empobrecido se mostraron más sensibles a las claves contextuales asociadas a la nicotina. En general, estos estudios confirman la hipótesis de que el ambiente es uno de los factores que contribuye a las diferencias individuales en la adicción, cese y recaída de la nicotina (Coolon y Cain, 2009). 
En la actualidad, la escasez de estudios sobre los efectos de la nicotina combinada con EE no permite determinar con exactitud los efectos de las manipulaciones ambientales en la respuesta conductual a esta droga, aunque datos previos sugieren la posible interacción entre el EE y el tratamiento con nicotina. Futuros estudios deberian investigar si variables experimentales como las diferencias en edad y/o cepa, variación en condiciones ambientales o periodo de exposición al EE pueden afectar a los posibles efectos neuroprotectores de esta droga de abuso. En nuestro laboratorio estamos actualmente abordando esta cuestión en el ámbito de diferentes proyectos de investigación financiados por el Plan Nacional de I+D+I y la Generalitat Valenciana (integrados en un proyecto Prometeo para grupos de excelencia). Nuestros resultados preliminares confirman que en ratones NMRI el ambiente enriquecido tiene efectos tanto fisiológicos (disminución de peso) como conductuales (disminución de la ansiedad y conducta exploratoria, menor actividad motora, mejor aprendizaje), similares a los descritos en otras cepas (Mesa-Gresa et al., 2010). Además, hemos observado que la nicotina disminuye la ansiedad y podría potenciar algunos de los efectos fisiológicos del ambiente enriquecido (MesaGresa, Pérez-Martínez, y Redolat, 2009).

\section{¿Cuál puede ser la dirección futura de la investiga- ción en los estudios que evalúan la relación entre ambiente enriquecido y adicción?}

Aunque los resultados de los estudios que han evaluado la interacción entre exposición a ambientes enriquecidos y desarrollo de la adicción a drogas de abuso resulta de enorme interés, debemos reconocer que todavía presentan evidentes limitaciones que deben ser superadas en futuras investigaciones. La mayoría de trabajos han utilizado periodos cortos de enriquecimiento y han comparado las condiciones de ambiente enriquecido no con ambientes estándar, sino con situaciones de aislamiento o empobrecimiento, lo que puede confundir los resultados y limitar su generalización (Laviola et al., 2008; Whitaker, Moy, Godfrey, Nielsen, Bellinger, y Bradfield, 2009). Tradicionalmente el ambiente enriquecido se ha establecido durante la adolescencia y únicamente en estudios recientes se ha evaluado el efecto del EE iniciado en la edad adulta en ratas adictas a diferentes sustancias de abuso (Puhl et al., 2012). Además, con frecuencia se obtienen resultados contradictorios que podrian explicarse por la utilización de diferentes especies, cepas, dosis o rutas de administración, así como por el uso de diversos procedimientos de EE. La principal dificultad para estudiar los beneficios y/o efectos producidos por el EE es la gran disparidad de paradigmas usados ya que, a pesar de que la base es similar, se pueden observar notables diferencias en cuanto al tamaño de las cajas, la composición, el número de sujetos, la complejidad de los objetos estimulantes y la frecuencia de cambio de los mismos (Bennett, McRae, Levy, y Frick, 2006; Whitaker et al., 2009). Una de las variables que más parecen influir en los resultados obtenidos y que resulta relevante en los estudios sobre adicción es la edad a la que se inicia la exposición al ambiente enriquecido (especialmente si se ini- cia en la adolescencia, etapa también critica en el establecimiento de la adicción) y durante cuánto tiempo se mantiene a los roedores en esa situación.

A pesar de estas limitaciones, los resultados disponibles hasta el momento confirman la hipótesis de que la adicción a diferentes drogas parece estar influida en gran medida por las condiciones ambientales. En este sentido, datos obtenidos en diferentes modelos animales apoyan la idea de que con el objetivo de prevenir la adicción sería muy importante proporcionar ambientes positivos y ampliamente enriquecidos, especialmente en etapas críticas del desarrollo (Solinas et al., 2010). Evidencia experimental reciente confirma que la exposición a ambientes complejos y enriquecidos podría resultar crucial para determinar el papel de las variables ambientales en la vulnerabilidad a la adicción y el desarroIlo de la resistencia ante algunas drogas de abuso como la cocaína (Laviola et al., 2008; Magalhaes et al., 2007), la heroína (El Rawas et al., 2009), el alcohol (Rueda et al., 2011) o la nicotina (Green et al., 2003; Hamilton y Kolb, 2005; Zhu et al., 2009).

Aunque los mecanismos neurobiológicos que subyacen a esta protección no se conocen totalmente, se están comenzando a proponer interesantes modelos teóricos. Los posibles efectos protectores del EE frente a la adicción podrían atribuirse a cambios hormonales, neurales, celulares y/o moleculares, por lo que la investigación de estas posibles alteraciones queda abierta a próximos estudios (Puhl et al., 2012). Parece estar claro que el EE ejerce importantes efectos sobre el cerebro a nivel estructural y funcional, centrándose la mayoría de los datos publicados en la memoria y el aprendizaje (Laviola et al, 2008). Son necesarios más estudios para evaluar con mayor detalle otras funciones conductuales y determinar cómo el ambiente enriquecido puede inducir cambios adaptativos cuando el cerebro se enfrenta a retos como la degeneración asociada el envejecimiento (Segovia et al., 2010), las alteraciones provocadas por el estrés crónico (Chen et al., 2010) o la exposición a sustancias de abuso como la nicotina (Redolat, Pérez-Martínez, Carrasco, y Mesa, 2009). También resultaría de gran interés diferenciar si los efectos beneficiosos del ambiente complejo se observan únicamente en comparación con las situaciones de "empobrecimiento ambiental" en las que en muchas ocasiones se mantiene a los animales en el laboratorio.

Las investigaciones preclínicas pueden contribuir a establecer más claramente qué factores individuales y ambientales contribuyen a la vulnerabilidad y/o resistencia a la adicción a determinadas sustancias. Estos estudios experimentales conllevan importantes implicaciones prácticas y terapéuticas ya que pueden ayudarnos a entender qué factores explicarian la observación frecuente de que no todos los sujetos que experimentan con la nicotina $u$ otras drogas se conviertan en adictos. Un mejor conocimiento de aspectos relacionados con la plasticidad (teniendo en cuenta cambios epigenéticos, cambios a nivel molecular y alteraciones en el circuito de recompensa) podría resultar útil para abordar nuevas aproximaciones al tratamiento (Foscarin, Rossi, y Carulli, 2011). 
Nuestra intención ha sido poner de manifiesto que los resultados obtenidos en los estudios preclínicos basados en ambientes enriquecidos pueden aportar información relevante en el ámbito de las adicciones a diversas drogas de abuso, lo que podría tener implicaciones tanto en modelos preclínicos como en la prevención y el tratamiento de la adicción en sujetos humanos. En este sentido, datos experimentales sugieren que los sujetos que se mantienen en ambientes estimulantes muestran una menor tasa de recaída tras superar una adicción, sugiriendo la importancia del mantenimiento de un estilo de vida más activo (García-Fernández et al., 2011). En conclusión, y a pesar de la gran complejidad ambiental e individual de los seres humanos y la dificultad en la extrapolación de los resultados obtenidos en animales, las investigaciones realizadas muestran que el paradigma del EE podría ser útil en el abordaje terapéutico de un problema de gran relevancia social como es la adicción a drogas de abuso.

\section{Agradecimientos}

Este trabajo ha sido posible por la financiación obtenida del Ministerio de Ciencia e Innovación y Plan E (PSI200910410) y de la Generalitat Valenciana (GVACOMP2010-273 y PROMETEO/2011/048).

\section{Conflicto de intereses}

Los autores del artículo manifiestan no tener ningún conflicto de intereses.

\section{Referencias}

Arndt, S. S., Laarakker, M. C., van Lith, H. A., van der Staay, F. J., Gieling, E., Salomons, A. R.,...Ohl, F. (2009). Individual housing of mice: impact on behaviour and stress responses. Physiology \& Behavior, 97, 385393. doi:10.1016/j.physbeh.2009.03.008.

Bardo, M. T., Bowling, S. L., Rowlett, J. K., Manderscheid, P., Buxton, S. T., y Dwoskin, L. P. (1995). Environmental enrichment attenuates locomotor sensitization, but not in vitro dopamine release, induced by amphetamine. Pharmacology, Biochemistry \& Behavior, 51, 397405. doi:10.1016/0091-3057(94)00413-D.

Bardo, M. T., Klebaur, J. E., Valone, J. M., y Deaton, C. (2001). Environmental enrichment decreases intravenous self-administration of amphetamine in female and male rats. Psychopharmacology, 155, 278-284. doi:10.1007/s002130100720.

Beauquis, J., Roig, P., De Nicola, A. F., y Saravia, F. (2010). Short-term environmental enrichment enhances adult neurogenesis, vascular network and dendritic complexity in the hippocampus of type 1 diabetic mice. PloS One, 5, e13993. doi:10.1371/journal.pone.0013993.

Bennett, J. C., McRae, P. A., Levy, L. J., y Frick, K. M. (2006). Long-term continuous, but not daily, environmental enrichment reduces spatial memory decline in aged male mice. Neurobiology of Learning and Memory, 85, 139-152. doi:10.1016/j.nlm.2005.09.003.

Bezard, E., Dovero, S., Belin, D., Duconger, S., Jackson-Lewis, V., Przedborski, S., ... Jaber, M. (2003). Enriched environment confers resistance to 1-methyl-4-phenyl-1,2,3,6-tetrahydropyridine and cocaine: Involvement of dopamine transporter and trophic factors. The Journal of Neuroscience: The Official Journal of the Society for Neuroscience, 23, 10999-11007.

Bowling, S.L., y Bardo, M.T. (1994). Locomotor and rewarding effects of amphetamine in enriched, social, and isolated reared rats. Pharmacology, Biochemistry \& Behavior, 48, 459-64. doi:10.1016/0091-3057(94)90553-3.

Brenes, J. C., Padilla, M., y Fornaguera, J. (2009). A detailed analysis of open-field habituation and behavioral and neurochemical antidepressant-like effects in postweaning enriched rats. Behavioural Brain Research, 197, 125-137. doi:10.1016/j.bbr.2008.08.014.

Burrows, E. L., McOmish, C. E., y Hannan, A. J. (2010). Gene-environment interactions and construct validity in preclinical models of psychiatric disorders. Progress in Neuro-Psychopharmacology \& Biological Psychiatry, 35, 1376-1382. doi:10.1016/j.pnpbp.2010.12.011.

Carvalho, A. F., Araujo, A. J., Farias, D. F., Rocha-Bezerra, L. C., y Cavalheiro, M. G. (2009). Development and reproductive performance of swiss mice in an enriched environment. Brazilian Journal of Biology, 69, 153-160.

Carroll, M. E., Anker, J. J., y Perry, J. L. (2009). Modeling risk factors for nicotine and other drug abuse in the preclinical laboratory. Drug and Alcohol Dependence, 104, S70-78. doi:10.1016/j. drugalcdep.2008.11.011.

Chauvet, C., Lardeux, V., Goldberg, S. R., Jaber, M., y Solinas, M. (2009). Environmental enrichment reduces cocaine seeking and reinstatement induced by cues and stress but not by cocaine. Neuropsychopharmacology: Official Publication of the American College of Neuropsychopharmacology, 34, 2767-2778. doi:10.1038/npp.2009.127.

Chen, Y., Mao, Y., Zhou, D., Hu, X., Wang, J., y Ma, Y. (2010). Environmental enrichment and chronic restraint stress in ICR mice: Effects on prepulse inhibition of startle and Y-maze spatial recognition memory. Behavioural Brain Research, 212, 49-55. doi:10.1016/j.bbr.2010.03.033.

Coolon, R. A., y Cain, M. E. (2009). Individual differences in response to novelty and the conditioned locomotor effects of nicotine. Behavioural Pharmacology, 20, 322-329. doi:10.1097/FBP. 0b013e32832f0176.

De Biasi, M., y Dani, J.A. (2011). Reward, addiction, withdrawal to nicotine. Annual Review of Neuroscience, 34: 105-30. doi:10.1146/ annurev-neuro-061010-113734.

Diamond, M. C. (2001). Response of the brain to enrichment. Anais Da Academia Brasileira De Ciencias, 73, 211-220. doi:10.1590/S000137652001000200006

El Rawas, R., Thiriet, N., Lardeux, V., Jaber, M., y Solinas, M. (2009). Environmental enrichment decreases the rewarding but not the activating effects of heroin. Psychopharmacology, 203, 561-570. doi:10.1007/s00213-008-1402-6.

El Rawas, R., Thiriet, N., Nader, J., Lardeux, V., Jaber, M., y Solinas, M. (2011). Early exposure to environmental enrichment alters the expression of genes of the endocannabinoid system. Brain Research, 1390, 80-89. doi:10.1016/j.brainres.2011.03.025. 
Foscarin, S., Rossi, F., y Carulli, D. (2011). Influence of the environment on adult CNS plasticity and repair. Cell Tissue Research, In press. doi:10.1007/s00441-011-1293-4.

Garcia-Fernández, G., Secades-Villa, R., Garcia-Rodríguez, O., AlvarezLópez, H., Fernández-Hermida, J.R., Fernández-Artamendi, S., y Higgins, S.T. (2011). Long-term benefits of adding incentives to the community reinforcement approach for cocaine dependence. European Addiction Research, 17, 139-145. doi:10.1159/000324848.

Green, T. A., Alibhai, I. N., Roybal, C. N., Winstanley, C. A., Theobald, D. E., Birnbaum, S. G., ... Nestler, E. J. (2010). Environmental enrichment produces a behavioral phenotype mediated by low cyclic adenosine monophosphate response element binding (CREB) activity in the nucleus accumbens. Biological Psychiatry, 67, 28-35. doi:10.1016/j.biopsych.2009.06.022.

Green, T. A., Cain, M. E., Thompson, M., y Bardo, M. T. (2003). Environmental enrichment decreases nicotine-induced hyperactivity in rats. Psychopharmacology, 170, 235-241. doi:10.1007/s00213-003-1538-3.

Green, T. A., Gehrke, B. J., y Bardo, M. T. (2002). Environmental enrichment decreases intravenous amphetamine selfadministration in rats: Dose-response functions for fixed- and progressive-ratio schedules. Psychopharmacology, 162, 373-378. doi:10.1007/s00213-002-1134-y.

Grimm, J. W., Osincup, D., Wells, B., Manaois, M., Fyall, A., Buse, C., y Harkness, J. H. (2008). Environmental enrichment attenuates cue-induced reinstatement of sucrose seeking in rats. Behavioural Pharmacology, 19, 777-785. doi:10.1097/FBP.0b013e32831c3b18.

Hamilton, D. A., y Kolb, B. (2005). Differential effects of nicotine and complex housing on subsequent experience-dependent structural plasticity in the nucleus accumbens. Behavioral Neuroscience, 119, 355-365. doi:10.1037/0735-7044.119.2.355.

Hannigan, J. H., O'leary-Moore, S. K., y Berman, R. F. (2007). Postnatal environmental or experiential amelioration of neurobehavioral effects of perinatal alcohol exposure in rats. Neuroscience and Biobehavioral Reviews, 31, 202-211. doi:10.1016/j.neubiorev.2006.06.019.

Hellemans, K. G., Nobrega, J. N., y Olmstead, M. C. (2005). Early environmental experience alters baseline and ethanol-induced cognitive impulsivity: Relationship to forebrain 5-HT1A receptor binding. Behavioural Brain Research, 159, 207-220. doi:10.1016/j. bbr.2004.10.018.

Ilin, Y., y Richter-Levin, G. (2009). Enriched environment experience overcomes learning deficits and depressive-like behavior induced by juvenile stress. PloS One, 4, e4329. doi:10.1371/journal.pone.0004329.

Kempermann, G., Fabel, K., Ehninger, D., Babu, H., Leal-Galicia, P., Garthe, A., y Wolf, S. A. (2010). Why and how physical activity promotes experience-induced brain plasticity. Frontiers in Neuroscience, 4, 189. doi:10.3389/fnins.2010.00189.

Laviola, G., Hannan, A. J., Macri, S., Solinas, M., y Jaber, M. (2008). Effects of enriched environment on animal models of neurodegenerative diseases and psychiatric disorders. Neurobiology of Disease, 31, 159168. doi:10.1016/j.nbd.2008.05.001.

Lee, V., y Hoaken, P. N. (2007). Cognition, emotion, and neurobiological development: Mediating the relation between maltreatment and aggression. Child Maltreatment, 12, 281-298. doi:10.1177/1077559507303778.

Lopez, M. F., Doremus-Fitzwater, T. L., y Becker, H. C. (2010). Chronic social isolation and chronic variable stress during early development induce later elevated ethanol intake in adult C57BL/6J mice. Alcohol (Fayetteville, N.Y.), 45, 355-364. doi:10.1016/j.alcohol.2010.08.017.

Magalhaes, A., Summavielle, T., Tavares, M. A., y de Sousa, L. (2007). Postnatal exposure to cocaine in rats housed in an enriched environment: Effects on social interactions. Human \& Experimental Toxicology, 26, 303-309. doi:10.1177/0960327106070458.

Mesa-Gresa, P., Pérez-Martínez, A., y Redolat, R. (2009, septiembre). Cambios emocionales inducidos por el enriquecimiento ambiental combinado con administración crónica de nicotina en ratones. Póster presentado en el XIII Congreso Sociedad Española de Neurociencia, Tarragona (España).

Mesa-Gresa, P., Pérez-Martínez, A., y Redolat, R. (2010, julio). A comparison of behavior in NMRI male mice in enriched environments and isolation-housing. Póster presentado en el $7^{\text {th }}$ FENS Forum of European Neuroscience, Amsterdam (Holanda).

Mesa-Gresa, P., y Moya-Albiol, L. (2011). Neurobiology of child abuse: The 'cycle of violence'. Revista De Neurología, 52, 489-503.

Meshi, D., Drew, M. R., Saxe, M., Ansorge, M. S., David, D., Santarelli, L., ... Hen, R.(2006). Hippocampal neurogenesis is not required for behavioral effects of environmental enrichment. Nature Neuroscience, 9, 729-731. doi:10.1038/nn1696.

Nithianantharajah, J., y Hannan, A. J. (2006). Enriched environments, experience-dependent plasticity and disorders of the nervous system. Nature Reviews.Neuroscience, 7, 697-709. doi:10.1038/ nrn1970.

Nithianantharajah, J., y Hannan, A. J. (2009). The neurobiology of brain and cognitive reserve: Mental and physical activity as modulators of brain disorders. Progress in Neurobiology, 89, 369-382. doi:10.1016/j.pneurobio.2009.10.001.

Nithianantharajah, J., y Hannan, A. J. (2011). Mechanisms mediating brain and cognitive reserve: Experience-dependent neuroprotection and functional compensation in animal models of neurodegenerative diseases. Progress in Neuro-Psychopharmacology \& Biological Psychiatry, 35, 331-339. doi:10.1016/j.pnpbp.2010.10.026.

Peña, Y., Prunell, M., Dimitsantos, V., Nadal, R., y Escorihuela, R. M. (2006). Environmental enrichment effects in social investigation in rats are gender dependent. Behavioural Brain Research, 174, 181187. doi:10.1016/j.bbr.2006.07.007.

Perry, J. L., Stairs, D. J., y Bardo, M. T. (2008). Impulsive choice and environmental enrichment: Effects of d-amphetamine and methylphenidate. Behavioural Brain Research, 193, 48-54. doi:10.1016/j.bbr.2008.04.019.

Pietropaolo, S., Feldon, J., Alleva, E., Cirulli, F., y Yee, B. K. (2006). The role of voluntary exercise in enriched rearing: $A$ behavioral analysis. Behavioral Neuroscience, 120, 787-803. doi:10.1037/07357044.120.4.787.

Pizzorusso, T., Berardi, N., y Maffei, L. (2007). A richness that cures. Neuron, 54, 508-510. doi:10.1016/j.neuron.2007.05.003.

Puhl, M.D., Blum, J.S., Acosta-Torres, S., y Grigson, P.S. (2012). Environmental enrichment protects against the acquisition of cocaine self-administration in adult male rats, but does not eliminate avoidance of a drug-associated saccharin cue. Behavioral Pharmacology, 23, 43-53. doi:10.1097/FBP.0b013e32834eb060.

Redolat, R., Perez-Martinez, A., Carrasco, M. C., y Mesa, P. (2009). Individual differences in novelty-seeking and behavioral responses to nicotine: A review of animal studies. Current Drug Abuse Reviews, 2, 230-242.

Redolat, R. y Mesa-Gresa, P. (2011). Potential benefits and limitations of enriched environments and cognitive activity on age-related 
behavioural decline. Current Topics in Behavioral Neuroscience, In press. doi:10.1007/7854_2011_134.

Rosenzweig, M. R. (1966). Environmental complexity, cerebral change, and behavior. The American Psychologist, 21, 321-332. doi:10.1037/h0023555

Rueda, A.V., Teixeira, A.M., Yonamine, M., y Camarini, R. (2011). Environmental enrichment blocks ethanol-induced locomotor sensitization and decreases BDNF levels in the prefrontal cortex in mice. Addiction Biology, In press. doi:10.1111/j.13691600.2011.00408.x.

Sale, A., Berardi, N., y Maffei, L. (2009). Enrich the environment to empower the brain. Trends in Neurosciences, 32, 233-239. doi:10.1016/j.tins.2008.12.004.

Schloesser, R. J., Lehmann, M., Martinowich, K., Manji, H. K., y Herkenham, M. (2010). Environmental enrichment requires adult neurogenesis to facilitate the recovery from psychosocial stress. Molecular Psychiatry, 15, 1152-1163. doi:10.1038/mp.2010.34.

Segovia, G., Del Arco, A., De Blas, M., Garrido, P., y Mora, F. (2010). Environmental enrichment increases the in vivo extracellular concentration of dopamine in the nucleus accumbens: A microdialysis study. Journal of Neural Transmission (Vienna, Austria: 1996), 117, 1123-1130. doi:10.1007/s00702-010-0447-y.

Smith, M. A., Chisholm, K. A., Bryant, P. A., Greene, J. L., McClean, J. M., Stoops, W. W., y Yancey, D. L. (2005). Social and environmental influences on opioid sensitivity in rats: Importance of an opioid's relative efficacy at the mu-receptor. Psychopharmacology, 181, 27-33.doi:10.1007/s00213-005-2218-2.

Smith, M.A., Bryant, P.A., y McClean, J.M. (2003). Social and environmental enrichment enhances sensitivity to the effects of kappa opioids: studies on antinociception, diuresis and conditioned place preference. Pharmacology, Biochemistry and Behavior, 200, 93-101. doi:10.1016/S0091-3057(03)00189-8.

Smith, M. A., Iordanou, J. C., Cohen, M. B., Cole, K. T., Gergans, S. R., Lyle, M. A., y Schmidt, K. T. (2009). Effects of environmental enrichment on sensitivity to cocaine in female rats: Importance of control rates of behavior. Behavioural Pharmacology, 20, 312-321. doi:10.1097/FBP.0b013e32832ec568.

Solinas, M., Thiriet, N., El Rawas, R., Lardeux, V., y Jaber, M. (2009). Environmental enrichment during early stages of life reduces the behavioral, neurochemical, and molecular effects of cocaine. Neuropsychopharmacology: Official Publication of the American College of Neuropsychopharmacology, 34, 1102-1111. doi:10.1038/ npp.2008.51.

Solinas, M., Chauvet, C., Thiriet, N., El Rawas, R., y Jaber, M. (2008). Reversal of cocaine addiction by environmental enrichment. Proceedings of the National Academy of Sciences of the United States of America, 105, 17145-17150. doi:10.1073/pnas.0806889105.
Solinas, M., Thiriet, N., Chauvet, C., y Jaber, M. (2010). Prevention and treatment of drug addiction by environmental enrichment. Progress in Neurobiology, 92, 572-592. doi:10.1016/j.pneurobio.2010.08.002.

Stairs, D. J., y Bardo, M. T. (2009). Neurobehavioral effects of environmental enrichment and drug abuse vulnerability. Pharmacology, Biochemistry, and Behavior, 92, 377-382. doi:10.1016/ j.pbb.2009.01.016.

Thiel, K. J., Pentkowski, N. S., Peartree, N. A., Painter, M. R., y Neisewander, J. L. (2010a). Environmental living conditions introduced during forced abstinence alter cocaine-seeking behavior and fos protein expression. Neuroscience, 171, 1187-1196. doi:10.1016/j. neuroscience.2010.10.001.

Thiel, K. J., Engelhardt, B., Hood, L. E., Peartree, N. A., y Neisewander, J. L. (2010b). The interactive effects of environmental enrichment and extinction interventions in attenuating cue-elicited cocaineseeking behavior in rats. Pharmacology, Biochemistry, and Behavior, 97, 595-602. doi:10.1016/j.pbb.2010.09.014.

Thiriet, N., Amar, L., Toussay, X., Lardeux, V., Ladenheim, B., Becker, K. G., ... Jaber, M. (2008). Environmental enrichment during adolescence regulates gene expression in the striatum of mice. Brain Research, 1222, 31-41. doi:10.1016/j.brainres.2008.05.030.

Thiriet, N., Gennequin, B., Lardeux, V., Chauvet, C., Decressac, M., Janet, T., ... Solinas, M. (2011). Environmental enrichment does not reduce the rewarding and neurotoxic effects of methamphetamine. Neurotoxicity Research, 19, 172-182. doi:10.1007/s12640-010-9158-2.

van Praag, H. (2009). Exercise and the brain: Something to chew on. Trends in Neurosciences, 32, 283-290. doi:10.1016/j. tins.2008.12.007.

van Praag, H., Kempermann, G., y Gage, F. H. (2000). Neural consequences of environmental enrichment. Nature Reviews. Neuroscience, 1, 191-198. doi:10.1038/35044558.

Whitaker, J., Moy, S. S., Godfrey, V., Nielsen, J., Bellinger, D., y Bradfield, J. (2009). Effects of cage size and enrichment on reproductive performance and behavior in C57BL/6Tac mice. Lab Animal, 38, 24-34. doi:10.1038/laban0109-24.

Wong, C.C., Mill, J., y Fernandes, C. (2011). Drugs and addiction: an introduction to epigenetics. Addiction, 106, 480-489. doi:10.1111/ j.1360-0443.2010.03321.x.

Zhu, J., Bardo, M. T., Green, T. A., Wedlund, P. J., y Dwoskin, L. P. (2007). Nicotine increases dopamine clearance in medial prefrontal cortex in rats raised in an enriched environment. Journal of Neurochemistry, 103, 2575-2588. doi:10.1111/j.14714159.2007.04951.x.

Zhu, S. W., Codita, A., Bogdanovic, N., Hjerling-Leffler, J., Ernfors, P., Winblad, B., ... Mohammed, A. H. (2009). Influence of environmental manipulation on exploratory behaviour in male BDNF knockout mice. Behavioural Brain Research, 197, 339-346. doi:10.1016/j. bbr.2008.09.032. 